\title{
Advanced Partial Run-time Reconfiguration on Spartan-6 FPGAs
}

\author{
Dirk Koch, Christian Beckhoff, and Jim Tørrison \\ Department of Informatics, University of Oslo, Norway \\ Email: \{dirk, christian\}@ recobus.de, jimtoer@ifi.uio.no
}

(Demonstration Paper)

\begin{abstract}
In this paper, we demonstrate systems based on Spartan-6 series FPGAs that provide full support for active partial run-time reconfiguration. We will summarize design factors for successfully applying run-time reconfiguration, reveal details on partial reconfiguration on Spartan-6 FPGAs, and introduce our easy to use design flow. In this flow, a module can multiple times be instantiated or even migrated to different systems without the need to physically reimplement such a module. The demo systems can host manifold different partial modules that each are capable to manipulate a video stream.
\end{abstract}

\section{INTRODUCTION}

Most publications, proposing to use partial run-time reconfiguration on FPGAs, aim to use smaller, and hence, cheaper and less power hungry devices to solve a particular problem, like for instance, [1] and [2]. However, these examples are based on devices of the Virtex-FPGA families from the vendor Xilinx. These FPGAs provide high-performance and high logic density FPGAs but at a higher monetary cost and with more power consumption as compared to the respectively Spartan series FPGAs. Furthermore, when following the design style proposed by the vendor Xilinx, a penalty in logic resources for providing an interface between the static system and the reconfigurable modules has to be considered. Moreover, this logic overhead comprises also a noticeable additional latency and may consequently harm performance [3].

The reason for this resource and latency overhead is that the latest vendor flow requires to pass each signal that crosses the border to a reconfigurable region through one extra look-up table (LUT). These LUT primitives acts as a connecting anchor (what Xilinx calles proxy logic) within the reconfigurable region and one side of the LUT is connected when routing the static part of the system and the respective other side of the LUT is connected when implementing a particular reconfigurable module. For example, an output signal of the static system will be routed to its associated anchor LUT input during the static implementation of the system. The final connection from the anchor output to the reconfigurable module is then set in a second implementation step that is based on an incremental design flow that takes the static system as a base. This second implementation step has to be repeated for each particular reconfigurable module.

Following the Xilinx vendor flow, the routing into a reconfigurable region cannot be constrained which has two consequences making this flow difficult to handle. First of all, when providing more than one reconfigurable region, modules cannot be relocated among the different regions as the anchor placement and routing is in general different in each region. A second consequence is that all partial modules have to be routed again when performing changes within the static part of the system, because the routing to the anchor LUT may differ. In other words, assuming a reconfigurable network processor with four regions and five different processing modules, as an example, it demands to reroute all $4 \times 5=20$ permutations on each modification of the static system. Note that this results also in 20 position dependent partial configuration bitstreams that have to be managed by the system at runtime.

\section{A. Successfully Applying Run-time Reconfiguration}

The basic prerequisite, a system must fulfill, is the existence of mutual exclusive functionality. Then, reconfiguration can be used to swap between different function blocks at runtime. In the easiest case, run-time reconfiguration can be seen as multiplexing between different modules, where changing the multiplexer state is carried out by a reconfiguration process. In addition to mutual exclusive functionality, it is important to consider the following issues:

Device support: Latest FPGAs provide typically a significant improvement in cost and power per logic function as compared to their predecessors. Consequently, runtime reconfiguration will only show a material benefit for commercial applications, if applied to the latest devices. Tool support: The design and verification of a run-time reconfigurable system should not take a significant higher effort than developing a system that is static only. Otherwise, the progress in silicon industry would overrun the potential benefit that might be achieved when applying partial run-time reconfiguration.

Efficiency: Applying run-time reconfiguration comes along with an overhead in terms of logic (e.g., for providing a configuration interface, for the communication, or because of fragmentation) and. im some cases, in terms of latency [4]. Consequently, efficient methods are required to implement run-time reconfigurable systems in order to achieve a material cost or power benefit as compared to a fully static only system.

All these issues should be solved prior to an implementing of a reconfigurable system. Unfortunately, as revealed afore, is the Xilinx vendor design flow still difficult to handle, if considering more than one reconfigurable region in a system. And moreover, the latest tools are not capable to implement 
reconfigurable systems for the recently introduced Spartan-6 FPGA family. So far, only small fractions of a design can be changed on these devices, like for example, logic functions within a LUT of the configuration of a clock generation block, when using the Xilinx vendor tools.

As an alternative, own tools have been developed and integrated into the synthesis and place \& route vendor framework. More information on the capabilities of our tools can be found in [4], [5]. The additional tools provide a floorplanner to generate advanced placement and routing constraints as well as a tool for the bitstream assembly. After introducing the specifics of Spartan-6 FPGAs regarding reconfiguration, we will sketch our design flow in Section III. After this, in Section IV, we will introduce two different demo system that are capable of hosting multiple times the same partial modules.

\section{INTERNALS OF XILINX SPARTAN-6 FPGAS}

In this section, we will summarize topics and differences of Spartan-6 FPGAs to other Xilinx FPGA families that have impact on partial run-time reconfiguration. Due to the $45 \mathrm{~nm}$ production process, Spartan-6 FPGAs provide high performance with practical clock speeds beyond $300 \mathrm{MHz}$, while significantly reducing the power per logic function at the same time. Spartan-6 FPGAs can be partially reconfigured via a 16-bit wide internal configuration port (ICAP) at a maximum specified speed of $200 \mathrm{MB} / \mathrm{s}$. These devices can continue operation during the reconfiguration process. Beside swapping modules, this capability might be used for scrubbing (in order to recover from single event upsets) or for bootstrapping, if the initial configuration time would be too long for a complete configuration process.

\section{A. The Spartan-6 Configuration Internals}

As Xilinx is not officially supporting partial reconfiguration within their tools for Spartan-6 FPGAs, the documentation on this feature is incomplete. In this section, we will reveal additional information on this topic. Most of the here presented information has been included into our tools and are not required to be understood by the application engineer.

Like all never Xilinx Virtex FPGAs, Spartan-6 devices can be configured in frames that span only a subpart of the fabric. Regardless to the total FPGA size, a frame contains parts of the configuration for a row of 16 configurable logic blocks (CLBs). Dedicated resources, including memory blocks and dedicated multipliers, follow this scheme and one frame contains parts of the configuration for four BRAMs or four DSP48 primitives. Opposed to previous Spartan-3 FPGAs it is not required to delete a full logic column prior to a configuration process [6].

The size of a frame is always 130 Byte and carries mainly the configuration for the primitives (e.g., 8 bytes per CLB). It requires to write $31(23 / 24)$ frames to the device for configuring all logic and routing information of one CLB (DSP48/BRAM) primitive. Note that the content of the memory block is stored in a separate region within the configuration bitstream. A frame has the full height of a clock region and clock buffers, located in the middle of each region, allow for each column to configure which global clock buffer is activated in the top and/or bottom half of a column.
As compared to Xilinx Virtex devices, there exist no separate configuration values for flip-flop init values and for reset values in Spartan-6 FPGAs and these values have been merged to one combined state within the configuration bitstream. Furthermore, there is also no capture primitive available that allows it any Virtex device to take a global snapshot of all flipflop values of the device while continuing device operation.

While we have not been able to configure Spartan-6 devices using the JTAG configuration port, full read and writes access to the fabric was possible using the internal configuration access port (ICAP) in Slave SelectMAP mode. As has been the case for previous Xilinx FPGAs, the bit order of each byte within the configuration bitstream has to be bytewise swapped before writing the data to the ICAP port.

\section{B. The Spartan-6 Logic Fabric}

The largest Spartan-6 devices provide only one fourth of the logic and almost eight times less local memory as compared to the latest Virtex- 6 devices. But not all applications demand the entire largest devices and partial run-time reconfiguration is an option to stuff more functionality into a given device.

A major advantage of the Spartan-6 logic architecture among the latest Virtex devices is that all I/O pins and dedicated hard-ip blocks (such as high-speed serial transceivers or PCI Express cores) are located at the borders of a particular device. This allows it to implement all peripherals (e.g., memory controllers) of the static system also at the borders while allowing to leave the majority of resources in a continuous large field for hosting reconfigurable modules. For systems hosting multiple reconfigurable modules at the same time, it is more beneficial to locate multiple modules into one large shared region instead of using an individual island for each particular module [4]. This reduces internal fragmentation that results from the fact that different modules have typically different resource requirements.

Using our regular structured communication architecture [5] allows it to relocate modules almost freely inside a reconfigurable region. This holds true, as long as the resource footprint of the modules match to the resources that are provided at the intended placement position within the reconfigurable region. For example, if a module posses block RAM resources, it can only be located to positions within the reconfigurable region that also provide RAM resources at exactly the same relative position within the module bounding box.

Besides the logic, RAM and dedicated multiplier primitives, there exist some further primitives for the clock network that state obstacles in the otherwise regular FPGA fabric. However, theses obstacles are relatively small (two CLBs) and only located at a few spots. Furthermore, it is possible to relocate a module possessing these obstacles to positions providing logic at that point.

Like in Virtex FPGAs, care has to be taken when instantiating distributive memory primitives, including distributed RAM or shift register primitives. If the vertical module placement or the definition of the reconfigurable region on the FPGA does not follow the 16 CLB tall configuration frame layout, the state of parts located above or below a module might get corrupted when reconfiguring the device. This is because 


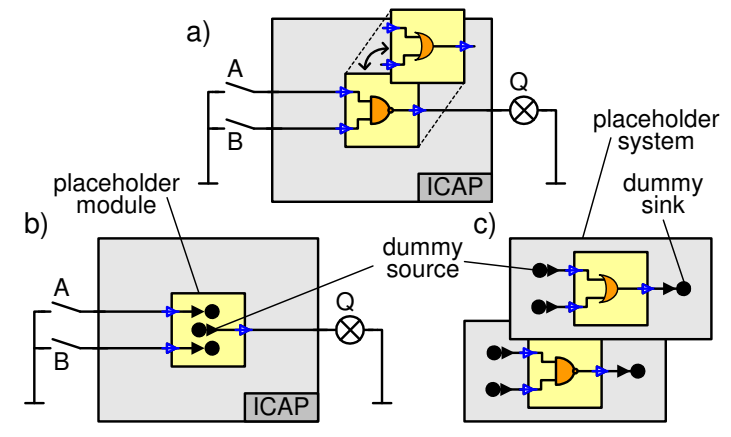

Fig. 1. a) Partially reconfigurable system composed of an implementation of b) a static system and c) independently generated modules.

the internal look-up table flip-flops of these primitives store a state that might get overwritten as the smallest atomic piece of configuration data comprises a full frame.

\section{The Spartan-6 Routing Fabric}

The routing fabric of Xilinx Virtex-6 FPGAs is basically identical to the Spartan-6 fabric. The main difference is that Spartan-6 FPGAs provide no long lines which are segmented wires that span over the full height or width of a device. Long lines are in particular useful for routing signals of the static system across a reconfigurable region.

A further issue to consider when implementing partially runtime reconfigurable systems on the latest Xilinx FPGAs is that these devices provide roughly the double amount of logic per CLB while possessing much less routing resources at the same time as compared to Virtex-II/Spartan-3 FPGAs [7]. This might harm performance or may even result in unroutable situations

\section{DESIGN FLOW}

We aim to implement the static system and the reconfigurable modules in completely isolated design steps. Then, all modules can be developed without the existence of the final static system. Moreover, modules are encapsulated and might be ported among different designs without any additional synthesis or place \& route step. This is possible by statically partitioning the routing resources of the FPGA into resources used for the top level communication and into resources that are used for the implementation of the static system or the reconfigurable modules.

In the easiest case, when only hosting exclusively one module in a reconfigurable region (island style), as illustrated in Figure 1, only the wires interfacing the reconfigurable modules have to be selected for the top level routing and constrained to certain fixed resources on the FPGA fabric. This means that all entity signals of a reconfigurable module, which are also the signals used for the connection in the top level routing of a static system, will be bound to predefined fixed wire resources. Considering an island reconfiguration style, only the wires that cross the border from or to a reconfigurable region have to be constrained. These constrained wires on the FPGA act like wrap connections on a printed circuit board (PCB).

\section{A. Implementing the Static System}

For defining a reconfigurable area within the static system and for constraining the interface wires that are allowed to cross the border for connecting a partial module, the user has to floorplan the system. This is supported by a comfortable GUI. For a selected reconfigurable region, our tools will generate constraints for the physical implementation of the static system that ensure that no logic and routing resources will be used within the selected reconfigurable area. For forcing the router to actually connect the entity signals to a fixed defined set of wires, we include a dummy sink to each input towards a reconfigurable module and respectively a dummy source for each module output, where each individual signal bit gets its own wire. See also Figure 1b).

The Xilinx vendor tools provide such prohibit constraints only for logic resources. We solved the remaining problem of constraining routing resources by generating blockers that occupy within a definable region all wire resources (or a selectable subset) that will then not be used by the Xilinx router for implementing the static system. For each interface signal, we leave a hole in the blocker such that the corresponding dummy sink or source can be connected. The dummies are LUT primitives that have to be instantiated as a partial placeholder module and that are placed inside the reconfigurable region. This step is also supported by our tools. With this blocking technique, we are able to bind a signal from an HDL description directly to a physical resource on the FPGA fabric.

Optionally, we can also leave wholes in the blocker for allowing the router to cross static signals in a controlled manner through the reconfigurable region. The released wires will be blocked during the implementation of the modules. This feature does not require any further user interaction.

\section{B. Implementing the Reconfigurable Modules}

The implementation of the reconfigurable modules follows the same idea of constraining than the one that has been applied to the static system. But this time, we constrain a particular module into an encapsulated region having the size and containing the same resource layout than the reserved reconfigurable area of the static system. This is achieved by prohibiting all logic and routing resources around the reconfigurable module, hence by putting a prohibiting fence around the reconfigurable module (see also Figure 1c) and Figure 2c)). For the routing, this is carried out with the help of a module blocker. Similar, as compared to the static system, where dummies have been used as a placeholder for the reconfigurable modules, we use now dummies as a placeholder for the static system. Consequently, the module implementation does not depend on the static system as it was implemented in in the last section.

For ensuring correct timing, a vendor supported MaxDelay constraint is applied to all interface signals that are connected to the dummy placeholders. By using different values for the static system and the partial modules, it is possible to freely balance the time slack between these two parts of the system.

It must be mentioned that neither the dummies used in the static system nor the ones that have been used for the different 
partial modules will constitute any additional logic or latency overhead, as it is the case following the Xilinx partial design flow. In our case, when loading a partial module into the static system, the original placeholders will be replaced.

\section{Hosting Multiple Modules in a Shared Region}

Besides the afore described island style that allows only one module to be placed exclusively in a reconfigurable region, our flow allows for placing multiple modules onto one shared region. This more advanced reconfiguration style can tremendously enhance the logic utilization in a partially reconfigurable system, because simple islands do not allow multiple smaller modules to replace one huge module.

This is possible by constraining the modules to provide compatible interfaces by binding the top level module signals to corresponding wires such that adjacent modules can directly communicate. In addition, modules can transparently route through signals between opposed borders and the static system can extend routing tracks within the reconfigurable area, as will be demonstrated in the next paragraph.

\section{DEMONSTRATION DESCRIPTION}

The implemented system provides a VGA background pattern generator that drives its output across two reconfigurable regions, as depicted in Figure 2. As can bee seen in the figure, the video stream is routed in a homogeneous manner such that the stream can be identically accessed at any possible module placement position. For this system, different reconfigurable overlay modules have been implemented that each access the video stream, manipulate the stream and send it further to the next overlay module or back into the static system. The set of modules includes a pong video game, which can be controlled using push buttons, object overlay modules, and different filters (convolution, edge detection, or color filtering).

We implemented the static system on two different boards: a SP605 board providing a Spartan-6 XC6SLX45T device and a SP601 board providing a XC6SLX16 FPGA. Despite having two fully different boards, the partial overlay modules have only been implemented once and identical partial modules are used in both static systems. Only the address fields in the module bitstreams, which specify the placement position on the FPGA, have been adopted. Furthermore, it is possible to instantiate each module multiple times. This is of interest for systems that should be able to adapt to different workload scenarios by instantiating more or less instances of the same accelerator dynamically at run-time.

Instead of providing a control CPU in the static system, we used a host PC that was in charge to manage the system and configurations have been sent via an UART, implemented on the entire target FPGA, directly to the internal configuration access port (ICAP). The results can be observed on the attached monitor. For the SP601 board, eight general purpose pins have been used to directly connect a VGA monitor. By using a passive resistor-based two bit DA converter per color, 64 different colors can be displayed.

\section{CONCLUSIONS}

In this paper, we revealed design factors for successfully implementing run-time reconfigurable systems and how this

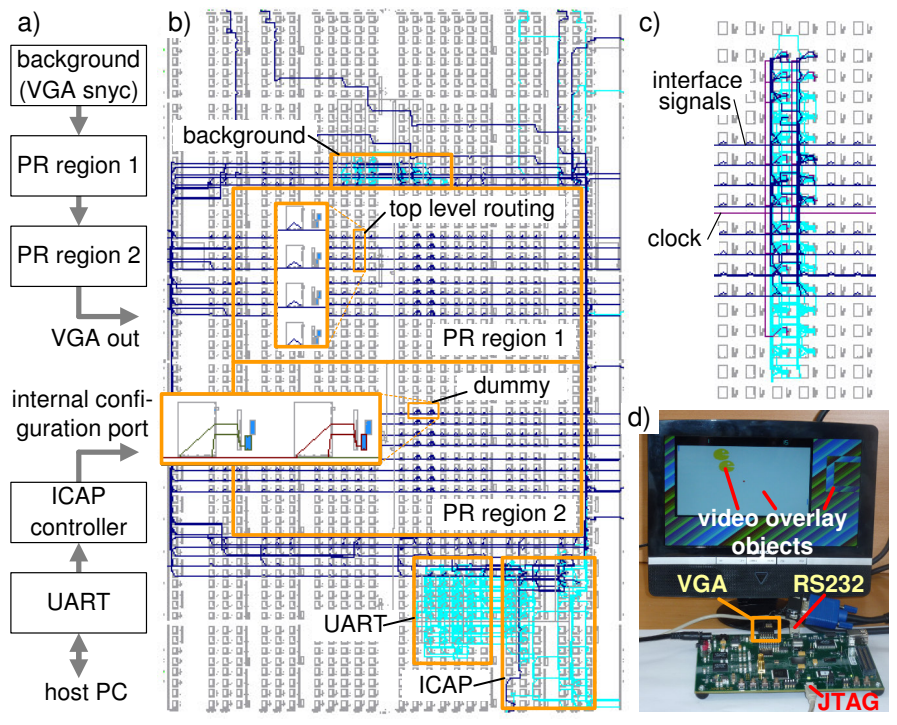

Fig. 2. a) Static design, b) FPGA-Editor screenshot of the static system providing the area for hosting partial modules, c) An encapsulated reconfigurable module, d) Demo system running on a Xilinx SP601 evaluation board.

can be applied to the latest generation Spartan-6 FPGAs. Not only supporting more cost efficient devices for implementing reconfigurable systems, we have also demonstrated a straighter design flow as compared to the Xilinx vendor tools. With our flow, real component based design methodology have been demonstrated that even allows the migration of modules among different systems. More information on our tools, the underlying theory, and the demo can be found on our project website: http://www.matnat.uio.no/forskning/prosjekter/crc/

\section{ACKNOWLEDGMENT}

This work is supported in part by the Norwegian Research Council under grant 191156V30

\section{REFERENCES}

[1] C. Claus, J. Zeppenfeld, F. Müller, and W. Stechele, "Using partial-runtime reconfigurable hardware to accelerate video processing in driver assistance system," in DATE '07: Proceedings of the conference on Design, automation and test in Europe. San Jose, CA, USA: EDA Consortium, 2007, pp. 498-503.

[2] E. El-Araby, I. Gonzalez, and T. El-Ghazawi, "Exploiting Partial Runtime Reconfiguration for High-Performance Reconfigurable Computing," ACM Trans. Reconfigurable Technol. Syst., vol. 1, no. 4, pp. 1-23, 2009.

[3] Xilinx Inc., "Partial Reconfiguration User Guide," Dec. 2009, rel 11.4.

[4] D. Koch, "Architectures, Methods, and Tools for Distributed Run-time Reconfigurable FPGA-based Systems," Ph.D. dissertation, University of Erlangen-Nuremberg, Germany, Erlangen, Dec. 2009.

[5] D. Koch, C. Beckhoff, and J. Teich, "ReCoBus-Builder - a Novel Too and Technique to Build Statically and Dynamically Reconfigurable Systems for FPGAs," in Proceedings of International Conference on FieldProgrammable Logic and Applications (FPL 08), Heidelberg, Germany, Sep. 2008, pp. 119-124.

[6] D. Koch, C. Beckhoff, and J. Teich, "A Communication Architecture for Complex Runtime Reconfigurable Systems and its Implementation on Spartan-3 FPGAs," in Proceedings of the 17th ACM/SIGDA International Symposium on Field-Programmable Gate Arrays (FPGA 2009). Monterey, California, USA: ACM, Feb. 2009, pp. 233-236.

[7] D. Koch and J. Torresen, "Routing Optimizations for Component-based System Design and Partial Run-time Reconfiguration on FPGAs," in IEEE International Conference on Field-Programmable Technology (FPT), 2010 . 